\title{
A proposed Novel Approach for Sentiment Analysis and Opinion Mining
}

\author{
Ravendra Ratan Singh Jandail \\ Computing Science and Engineering, Galgotias University, India
}

\begin{abstract}
as the people are being dependent on internet the requirement of user view analysis is increasing exponentially. Customer posts their experience and opinion about the product policy and services. But, because of the massive volume of reviews, customers can't read all reviews. In order to solve this problem, a lot of research is being carried out in Opinion Mining. In order to solve this problem, a lot of research is being carried out in Opinion Mining. Through the Opinion Mining, we can know about contents of whole product reviews, Blogs are websites that allow one or more individuals to write about things they want to share with other The valuable data contained in posts from a large number of users across geographic, demographic and cultural boundaries provide a rich data source not only for commercial exploitation but also for psychological \& sociopolitical research. This paper tries to demonstrate the plausibility of the idea through our clustering and classifying opinion mining experiment on analysis of blog posts on recent product policy and services reviews. We are proposing a Nobel approach for analyzing the Review for the customer opinion.
\end{abstract}

\section{INTRODUCTION}

Human opinion and perception always be the part of decision making either in the historical days by the Ruler, for employing any scheme and in some critical situation for their kingdom or in now days by any organization or country to know about its product, policy and services. Opinion Mining is a process, used for automatic extraction of knowledge from the opinion of others bout some particular topic or problem. Human perception and user opinion has greater potential for knowledge discovery and decision support. The goal of $\mathrm{OM}$ is to make computer able to recognize and express emotions. A thought, view, or attitude based on emotion instead of reason is called sentiment. Thus OM is also referred as sentiment analysis. During decision making process most of us get help from others. It is a natural phenomenon that good decision can be taken on the basis of opinion of others. Automatic detection of emotions in texts is becoming increasingly important from an applicative point of view. Survey, blogs and review site are used to collect customer opinion about products to get the knowledge about the reputation of the company in the market [1]. Now day's social media is the best tool to know about the

People opinion, advice, comment, complement and their perception about any product policy and services. We are utilizing social media for the manufacturer and consumer, service provider and user, policies and its effects. 
Social media has demonstrated itself to be a proven source of information towards the marketing of products. This unique source of data provides a rapid means of customer feedback that is used to support a number of business areas. Social media can provide immediate feedback in minutes and as such it represents a new challenge in communication between a customer and business. One can use social media to gauge customer response, in the form of what they like (or don't like), along with associated details. This can support many facets of a business including product development, customer service or marketing. Thus, it is important to utilize social media to avoid any shifts in brand image and corporate reputations when certain issues are not handled early and effectively. By leveraging such channels appropriately, the customer relationship can be strengthened, increasing value in all aspects of a company's business [2]. As The Lord Krishna said in our BHAGVAT GEETA that every relation of this universe is based on some needs and while the need is fulfilled the relation will be sweet but if the chain of need braked then the relation will also be destroy itself. So we are trying providing "not, what the manufacturer wants to develop?" But "What the consumer wants?" not "what the services is available?" but "what the quality of services user wants" not "what policies are applied?" but "how the policies should be for people?" by utilizing the social media blogs and reviews

\section{DEFINATION}

Many definition of sentiment analysis and opinion mining has been provided by different authors' publisher and writer but the most appropriate is that "Sentiment analysis is the way of Analyzing the social, psychological, philosophical, behavioral perception and opinion of a Person, people or group in a specific situation and condition about any

- Product

- Services

- Event

- Thought

- Person or organization

- Policy

- Weather and

Particular situation"

\section{III.DIFFERENT LEVEL OF ANALYSIS}

\section{A. Document level}

In this approaches whole document is considers as a single entity and the analysis approaches in applied on the whole document. The result generated in document level sometimes not appropriate

\section{B. Sentence level}

In the sentence level approaches every sentence is considered as an entity and analysis approaches is applied on individual sentence then their result is summarized to provide the overall result of the document. It is known as Clause level analysis 


\section{Entity and Aspect level}

It is a feature based approaches in this approaches we Considered positive and negative sentiments for the Quality of features. This approaches is based on the Opinion mining and summarization

\section{IV- ISSUES IN SENTIMENT MINING}

There are six kind of issues in the sentiment analysis but sometimes the main problem is occur while opinion suitable for user group became harmful for the manufacturer or vice versus which are elaborate by Bing Liu in his book "Sentiment Analysis and Opinion Mining".

a. A positive or negative sentiment world may have their opposite meaning in a particular domain so it is hard to predict by its keyword meaning.

Ex- the picture quality of this camera is high and the resuming time of this camera is also high. In this sentence the first HIGH is showing the positive sentiment for the camera but the second high is showing the negative sentiment for the camera.

b. Interrogative Sentence An interrogative sentence may not have neither positive nor negative sentiment but the key word used in the opinion may be positive or negative.

Ex1. Can anybody tell me is HCL a good laptop for an engineering student for multicore programming?

2. What are the good and bad functionality of hero-Honda motorcycle?

Such kind of sentences don't have any positive or negative sentiment for analysis but the keyword used in such sentences have its precious and valuable meaning for analyzing any sentences.

\section{c. Sarcastic Sentences}

Few sentences in the form of jocks may violate the meaning of the whole sentences such kind of sentence need a power full attention toward the keywords and sentences. These funny sentences not only violet the sentence of a particular sentence but also destroy the value of the whole document. Ex- 1. What a Great Car? It stopped Working in 10 days. Who is going to purchase such beautiful car?

4. Sentiment without sentiment words sometimes sentiments does not use any sentiment words like good, better, best, worst ,bad etc. but the sentences may have its positive or negative feedback about the product, services and policies. Ex- this car consume lot diesel from Delhi to Chandigarh then the other one. This sentence showing the negative sentiment but it is not using the any sentiment word.

\section{Natural language Issues Change Place to Place}

Motorola word can be used as motto, Lamia word can be used as Lummi. Such kind of sentences changes the meaning of the issue and sentences. 
International Journal of UbiComp (IJU), Vol.5, No.1/2, April 2014

6. Conditional sentences conditional sentences are also an issue in Sentiment mining conditional sentences is also creating the same problem like interrogative sentences.

Ex- If the picture quality of this camera is good I will buy it.

\section{Author and Reader understanding point (person to person varying)}

Dollar price is increasing with respect to Indian rupee.

This document have both the positive and negative meaning and its value is varying from person to person. This sentence has the positive sentiment for the Exporter while this same sentence has the negative sentiment for the importers.

8. Spam Reviews Spam sentiments are those sentiments which are posted by the opposite or competitor organization for increasing their product value or their organization value among the users. Some politician may use the same spam review to just for their publicity.

\section{APPLICATION OF SENTIMENT ANALYSIS}

Early applications of sentiment analysis focused on classifying movie reviews or product reviews as positive or negative or identifying positive and negative sentences, but many recent applications involve opinion mining in ways that require a more detailed analysis of the sentiment expressed in texts. One such application is to use opinion mining to determine areas of a product that need to be improved by summarizing product reviews to see what parts of the product are generally considered good or bad by users. Another application requiring a more detailed analysis of sentiment is to understand where political writers fall on the political spectrum, something that can only be done by looking at support or opposition to specific policies A couple of others applications, like allowing politicians who want a better understanding of how their constituents view different issues, or predicting stock prices based on opinions that people have about the companies and resources involved the marketplace, can similarly take advantage of structured representations of opinion. These applications can be tackled with a structured approach to opinion extraction [4].One specific application of sentiment in NLP that can be used for this purpose is sentiment analysis. It can be used to identify and extract subjective information from the information source collected. With all these processes and methods, it is possible to build a system which can extract application dependent information, process it and produce data which can be used for studying and deductions based on the information retrieved [5].Much of the current opinion mining research has focused on business and e-commerce applications, such as product reviews and movie ratings. Little research has tried to understand opinions in the social and geopolitical context [6].

Applications to Review-Related Websites Applications as a Sub-Component Technology Applications in Business and Government Intelligence Applications across Different Domains [7] 
International Journal of UbiComp (IJU), Vol.5, No.1/2, April 2014

\section{MACHINE LEARNING TECHNIQUE USED CLASSIFICATION OF TEXT IN SENTIMENT MINING}

Since the beginning of this century, several researchers have studied the implementation of machine learning technique to capture sentiments from online reviews using this technique, most researchers treated opinion mining as a text classification problem. Conventionally, this method involves the creation of a model using statistical technique such as Support Vector Machine

(SVM) and Naïve Bayes (NB). The sentiment of a new sentence or document is predicted using a particular statistical model. The results of opinion mining were compared using three statistical models. They concluded that even though these techniques produce good result in a normal text classification problem, it is not the same case with opinion mining [8]

Engineers and computer scientists use machine-learning techniques for automatic affect classification from video, voice, text, and physiology. Psychologists combine the long tradition of emotion research with their own discourse, models, and methods. Opinion mining and sentiment analysis are inextricably bound to the affective sciences that attempt to understand human emotions. Affect-sensitive systems and psychological emotion research must develop together [9].

The most widely used machine learning techniques are following

1. Naive bayes classification

2. Maximum entropy rule

3. Decision induction tree

4. Neutral network

5. Probability latent semantic

6. Latent Dirichlet Allocation

\section{PROBLEM DEFINITION AND OUR PROPOSAL}

The primary question for sentiment analysis is how to map a tweet to a correct emotion, which user tried to express. The first problem is unstructured, ungrammatical text. Since tweet messages are restricted to 140 characters length, users may have a propensity to use abbreviations, slangs, or emoticons to shorten the text. This issue can lead to unusual messages.

The second problem is the fact that tweet messages are not always correct. During fast typing, or using mobile phones as input device, user may have mistyped text and make the analysis step harder.

The third problem is ambiguity. Due to the small amount of information, it is difficult to identify the corresponding objects of interest. For example: "Apple" can either be a laptop brand or a fruit.

The fourth problem concerns which concrete emotion to focus on analyzing since human emotion is very diverse. 
International Journal of UbiComp (IJU), Vol.5, No.1/2, April 2014

The difficulty lies in the fact that there could be mixed opinions in a document, and with the creative nature of natural languages, people may express the same opinion in vastly different ways, sometimes without using any opinion words:

We are also try to overcome the issue related to sentiment mining as we discussed earlier $\mathrm{i}$ the section 2 .

\section{OUR METHODOLOGY}

Generally approached are used for Sentence level, Document level, or feature level but our approaches will work for all the three above mentioned levels. First we Split the document in the sentences then every sentence will classify according to its problem domain, if sentence is not belongs to any domain then it will be consider as the normal sentence.

No of available keyword in any sentence will be the weight age of that particular sentence.

In case of product, the product will be classified in its attributes and on the basis of keyword available for a particular attributes we give a weight age -5 to +5 . In this way every attribute will be weighted -5 to +5 . After all, the average of the all attributes will be the average of the weight age of the overall product. In this way not even we can summaries about the product but also the individual attributes available in the product.

For Example - A Mobile phone have attributes like, weight, picture quality, Resolution, touchpad, keypad, voice quality, music quality, Bluetooth, etc. By our approach we can predict about a particular attribute and the overall product according to the user perception.

\section{A. FLOW CHART}

Flowchart is showing the working of our designed algorithm for People product review Analysis. Flowchart is showing top down approach for methodologies. 
International Journal of UbiComp (IJU), Vol.5, No.1/2, April 2014

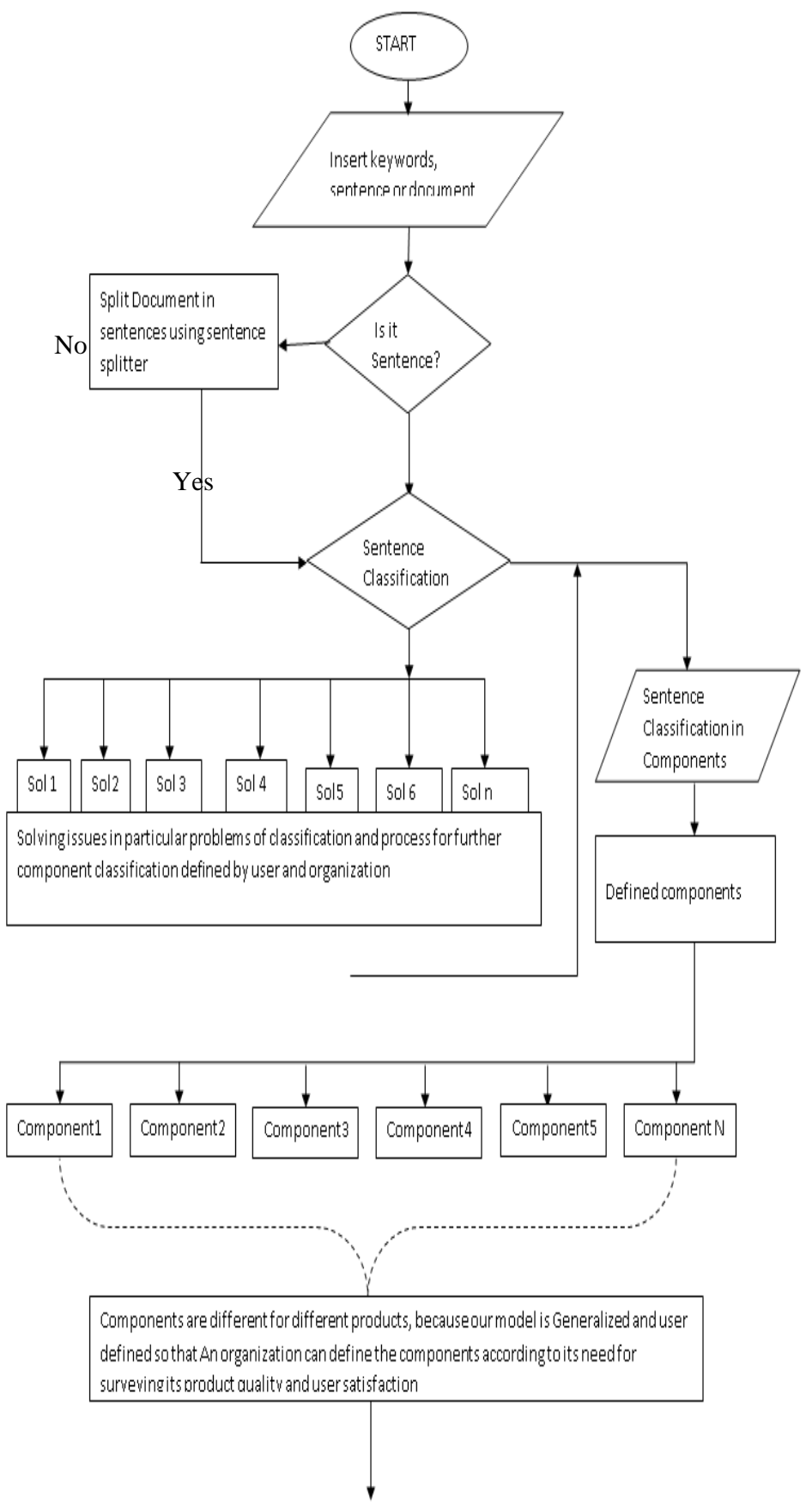




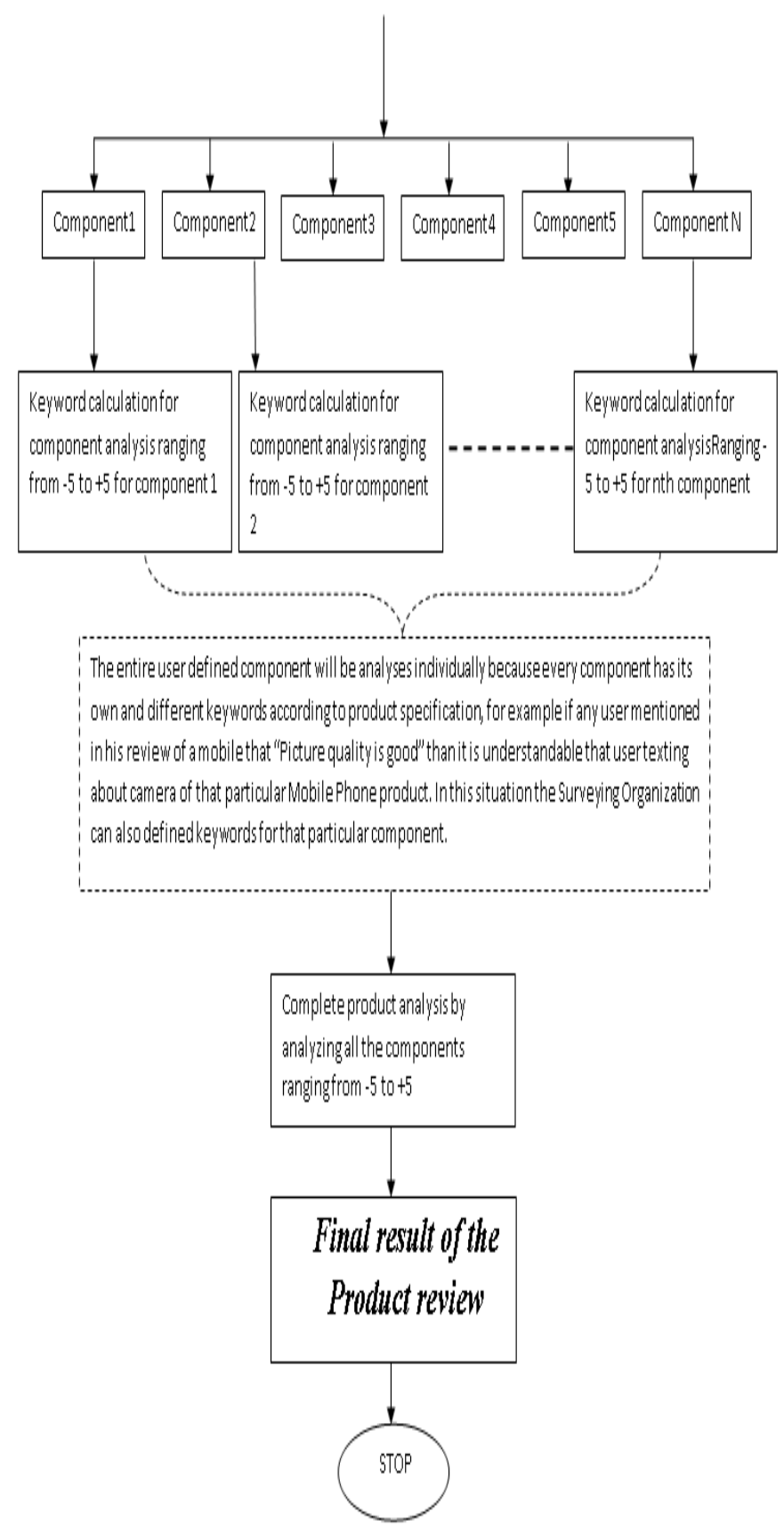

We are Using Support Vector Machine Learning Technique for text classification in its classes and predefined Feature. If we take the Example of Cell Phone than Cell phone is the class and Camera, Sound, Keypad, Battery are its predefine feature of the class cell phone. 


\section{SENTIMENT WORD DICTONARY-}

We designed a Dictionary of 576 sentiment words after analyzing the more than 5000 reviews of the mobile phone collected from www.amazon.com, www.areena.com and twitter. We have assigned weight for every sentiment word from -5 to +5 as its importance by user sentiment for example "the Sound of this speaker is heavy and this camera is also heavy to carry". In this sentence the Word heavy is used for both Camera and the Speaker, everyone do understand that the Word "Heavy" is positive for the Speaker but this word is negative for the Camera Weight. So we assigned rating for every word as its value for ever sentiment.

\section{SENTIMENT CALCULATION}

We are able to analyses the total text in its positive and negative polarity for the particular feature.

$$
\begin{aligned}
& \mathrm{S}(\mathrm{W}+\mathrm{ve})=\text { Set of Positive Sentiment words } \\
& \mathrm{S}(\mathrm{W}-\mathrm{ve})=\text { Set of Negative Sentiment words } \\
& \text { For } \mathrm{N}^{\text {th }} \text { Feature }- \\
& \mathrm{S}\left(\mathrm{W}_{+}\right)=(\mathrm{W} 1+\mathrm{W} 2+\mathrm{W} 3+\ldots \ldots . \mathrm{Wn}) \text { Set of } \\
& \quad \begin{aligned}
& \text { Positive Sentiment Words } \\
& \mathrm{S}\left(\mathrm{W}_{-}\right)=(\mathrm{W} 1+\mathrm{W} 2+\mathrm{W} 3+\ldots \ldots . . \mathrm{Wn}) \text { Set of } \\
& \text { Negative Sentiment Words }
\end{aligned}
\end{aligned}
$$

First of all we calculate the negative and positive polarity every sentiment by calculating its probability. Then we shall calculate the mutual information of its positive or negative sentiment.

After calculating the overall polarity of the individual feature we shall calculate the Mutual information for every feature for its final product review Analysis.

\section{RESULT}

Initially we obtained these results for the mobile phone only, we include 16 most usable feature and their related keywords for the mobile phone. We will also apply this for movie sentiments and other most usable products. We are designing dictionary and keywords for the specific products like camera and washing machine. 
International Journal of UbiComp (IJU), Vol.5, No.1/2, April 2014

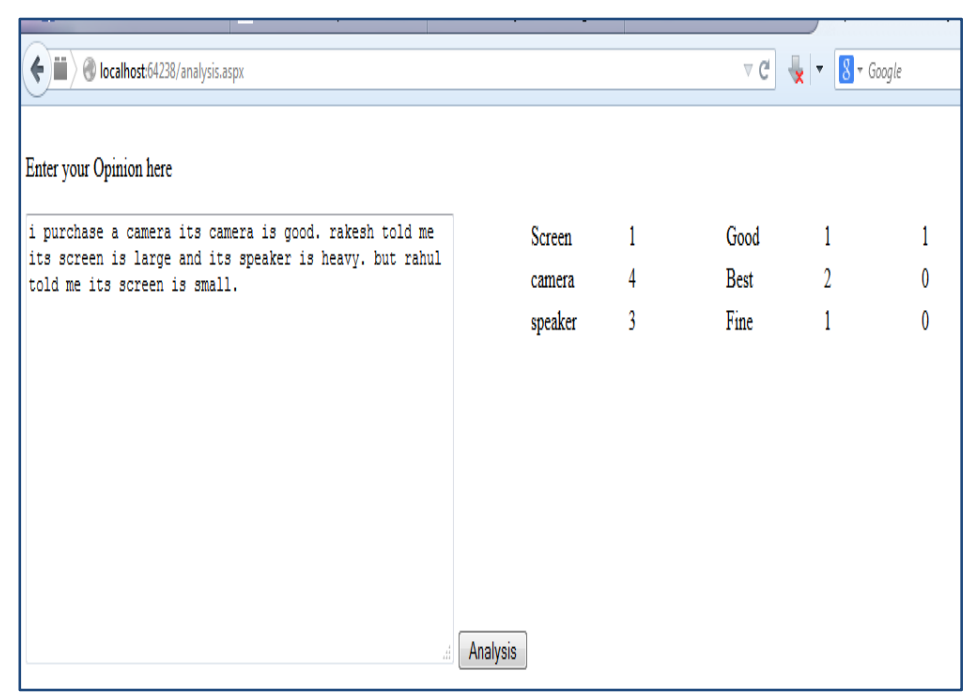

\section{FUTURE WORK}

Initially we are applying our approach on product usability analysis .It will classify users review in its components and all components usability is decide by keywords base learning. In future we will proceed it for policy and services. We believe that we will provide better result for the Product usability analysis. After completing this phase we shall apply it for the situations also.

\section{References-}

[1] Khairullah Khan, BaharumB.Baharudin, Aurangzeb Khan, Fazal-e-Malik, Mining Opinion from text Documents: A Survey, 3rd IEEE International Conference on Digital Ecosystems and Technologie, 2009

[2] David Alfred Ostrowski, Sentiment Mining within Social Media forTopic Identification ,IEEE Fourth International Conference on Semantic Computing 2010

[3] ANA SUFIAN ,RANJITH ANANTHARAMAN ,Social Media Data Mining and Inference system based on Sentiment Analysis 2011

[4] KENNETH BLOOM,Sentiment Analysis Based On Appraisal Theory And Functional Local Grammar 2011.

[5] ANA SUFIAN ,RANJITH ANANTHARAMAN, Social Media Data Mining and Inference system based on Sentiment Analysis 2011

[6] Hsinchun Chen and David Zimbra,AI and Opinion Mining, ,IeeeInTeLLIGenTSySTeMS 2010

[7] Bo Pang and Lillian Lee Opinion Mining and Sentiment Analysis, Foundations and TrendsR inInformation Retrieval 2008

[8] NorlelaSamsudin, MazidahPuteh, Abdul RazakHamdan, MohdZakree Ahmad Nazri, Is Artificial Immune System Suitable for OpinionMining? 4th Conference on Data Mining and Optimization (DMO)

, Langkawi, Malaysia 02-04 September 2012

[9] Erik Cambria, BjörnSchuller, Yunqing Xia, Catherine Havasi, Published by the IEEE Computer Society 2013

[10] ThanhThung ,evaluation of natural language Processing technique for Sentiment Analysis , 2012 